\title{
Fragmented endocardial signals and early afterdepolarizations during torsades de pointes tachycardia
}

\author{
Guo-Liang Li ${ }^{1,2 *}$, Ardan M. Saguner ${ }^{3 *}$, Guy H. Fontaine ${ }^{2}$, Robert Frank ${ }^{2}$ \\ ${ }^{1}$ Arrhythmia Unit, Department of Cardiovascular Medicine, First Affiliated Hospital of Xi'an \\ Jiaotong University, Xi'an, Shaanxi, P.R. China \\ ${ }^{2}$ Institut de Cardiologie, Unité de Rythmologie, Groupe Hospitalier Pitié-Salpêtrière, Paris, France \\ ${ }^{3}$ Department of Cardiology, University Heart Center Zurich, Switzerland
}

\begin{abstract}
Background: Bradycardia-induced torsade de pointes (TdP) tachycardia in patients with spontaneous high-degree atrioventricular block $(A V B)$ is common. The aim of this study was to analyze endocardial recordings during TdP in spontaneous high-degree AVB in humans to better understand the electrophysiological mechanisms underlying this phenomenon.

Methods: The study group consisted of 5 patients with typical episodes of TdP during spontaneous high-degree AVB. A standard (USCI) temporary bipolar endocardial catheter positioned at the apex of the right ventricle $(R V)$ and bipolar chest leads from two precordial leads V1 and V4 were used to record the tracings during $T d P$.

Results: The presence of a wide spectrum of fragmentations was noted on endocardial electrograms (EGMs), which were invisible on the surface electrocardiogram (ECG) tracing. Endocardial signals indicated that TdP started in the proximity of the RV apex, since the local EGM began prior to the QRS complex on the surface ECG. Early afterdepolarizations (EADs) were observed in 2 out of 5 cases confirming a common opinion about the mechanism of TdP. However, this phenomenon was not observed in 3 other patients suggesting that the arrhythmia was the result of a different mechanism originating in proximity to the $R V$ apex.

Conclusions: This work demonstrated early endocardial signals in the RV apex during TdP associated with high-degree AVB in humans, and exhibits a spectrum of fragmented signals in this area occurring on a single or multiple beats. These fragmentations indicate areas of poor conduction and various degrees of intramyocardial block, and therefore a new mechanism of TdP tachycardia in some patients with spontaneous high-degree AVB. (Cardiol J 2020; 27, 1: 54-61)
\end{abstract}

Key words: early afterdepolarizations, torsades de pointes, electrocardiogram, atrioventricular block, endocardial recordings

\section{Introduction}

The exact mechanism of torsades de pointes $(\mathrm{TdP})$ tachycardia in high-degree atrioventricular block (AVB) and long QT syndrome (LQTS) has been puzzling electrophysiologists over the past decades [1-5]. This syndrome or its variants are a rare but important cause of sudden death in young people with a genetically determined form of LQTS $[6,7]$. The length of the QT interval

Address for correspondence: Dr. Robert Frank, Institut de Cardiologie, Unité de Rythmologie, Groupe Hospitalier Pitié-Salpêtrière, 47-83 Boulevard de l'Hôpital, 75651, Paris, France, e-mail: robertfrank@sfr.fr

Received: 4.04.2018

Accepted: 8.07.2018

* Shared first authors. 
is directly related to risk stratification of these patients modulated by genetic factors [8]. TdP is also a major concern to the pharmaceutical industry for the development of cardiac and noncardiac drugs [9]. In these cases the role of early afterdepolarizations (EAD) has been suggested as their main trigger [10].

Therefore, it is important to understand the basic mechanisms of this form of polymorphic ventricular tachycardia (VT), originally denominated in patients with high-degree AVB in 1966. The descriptive term of "torsade de pointes" was chosen to describe the fact that QRS complexes seem to twist around the isoelectric line [11]. A German team reproduced this arrhythmia experimentally giving credit to this original mechanism of two alternating ventricular foci [12]. The aim of this study is to report on right ventricular (RV) endocardial recordings in vivo in humans presenting unexpected signals for the first time during high-degree AVB-induced TdP or its impending premature ventricular contractions (PVCs), couplets, triplets etc., which are considered as the minor equivalents of TdP. These events produce successive short-long intervals until the initiation of TdP.

\section{Methods}

This is a retrospective study. When G.H.F. carried out these studies between 1965 and 1970, there were no ethical standards of a responsible committee on human experimentation (institutional or regional). This investigation was approved by the chief of cardiology as there was a clinical need to study these patients. In those times it was important to verify that polymorphic non sustained VT (early name for TdP) [13] could be prevented by ventricular pacing in non ischemic patients with AVB-induced bradycardia, which is not possible in polymorphic VT due to myocardial ischemia. An oral informed consent by the patient was obtained before the procedure.

The population consisted of 5 patients exhibiting TdP resulting from spontaneous high-degree AVB. Most of these patients were waiting for pacemaker (PM) implantation, replacement or repair at a time when these devices were not readily available and were expensive. Therefore, they had a temporary pacing system with an endocardial electrode and external PM. A standard temporary bipolar pacing endocardial catheter (USCI, Billerica, MA USA) was positioned at the apex of the RV. A bipolar electrocardiogram (ECG) was derived from two precordial electrodes placed at the V1 and V4 positions. In fact, this bipolar recording extracts information mostly from the RV since both electrodes are put in regular place in the parasternal intercostal space (V1) and V4, which is mostly covering the RV. They were connected to a 3A9 differential amplifier with a band pass from direct current to $-1 \mathrm{Mhz}$ with an industrial oscilloscope (Tektronix 361A Portland, Oregon, USA) equipped with a phosphor cathodic tube and a 2B67 time base, in those times when specific electrophysiological equipment was not available. The amplifier output was connected to a tape recorder (Ampex SP 300, Redwood City, CA, USA). The tapes were subsequently reviewed with an oscilloscope (Tektronix 561A-3A72) to select episodes of TdP, which were printed on a two-channel industrial ink pressure recorder (Clevite Brush 220 Chart Recorder Cleveland, OH, USA). An external defibrillator was readily available before the disconnection of the endocardial lead. Preliminary findings from this patient population have been previously published by the present group [14] The current study extends those findings and further elucidates on the pathophysiology of TdP tachycardia in high degree AVB.

\section{Results}

\section{Study population}

Patients were $>65$ years old; $80 \%$ were females. They had no sign of coronary artery disease, but presented with AVB type II Mobitz or AVB III. Most of them had well tolerated short episodes of TdP (less than $5 \mathrm{~s}$ ) preceded by PVCs, couplets, triplets etc.

\section{Tracings}

Tracings will be presented according to their increasing complexity.

Variable patterns of ventricular activation until reorganization of ventricular activation leading to the termination of most $\mathrm{TdP}$ episodes were noted on the endocardial tracings and surface ECG. The recordings allow to analyze the different apical endocardial characteristics of the initiating beat, and of the following ones.

Figure 1 shows the escape junctional rhythm followed by a ventricular triplet. The initiating endocardial signal consists of two consecutive negative high amplitude contiguous sharp potentials at the beginning of the triplet. The first sharp potential starts at the same time as the surface ECG tracing. This tracing shows no other fragmentation 




Figure 1. Two consecutive negative high amplitude contiguous sharp potentials (red arrowheads) were observed at the beginning of a triplet. There is no visible early afterdepolarization (EAD) or EAD-like pattern.

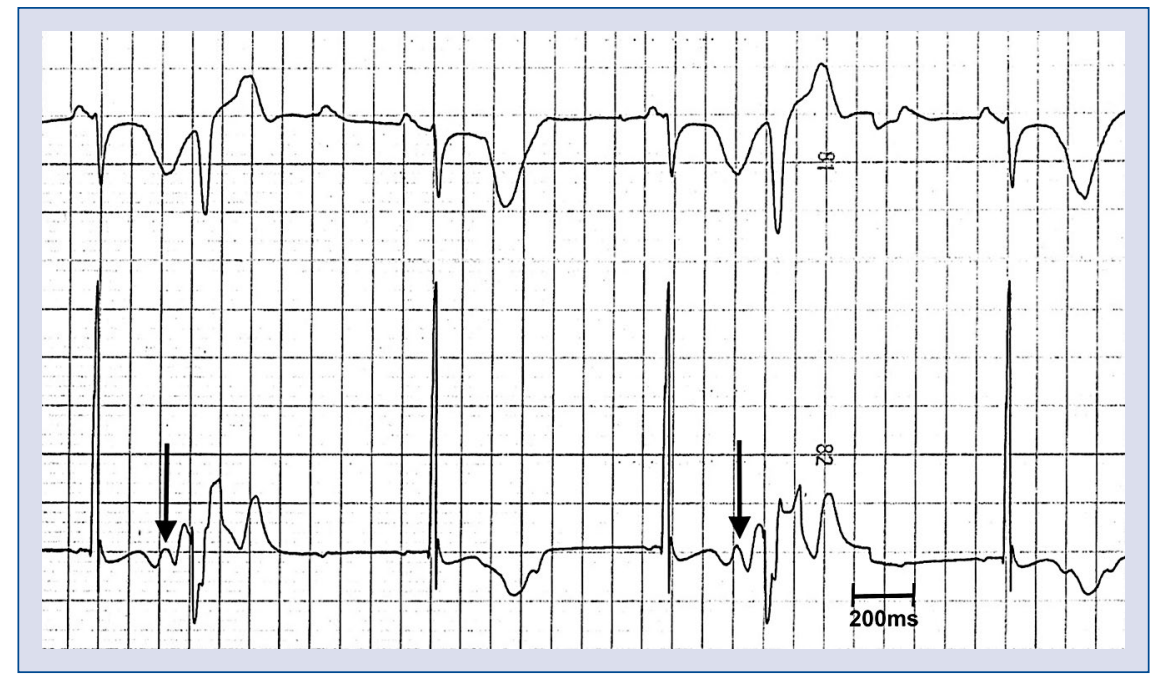

Figure 2. On this electrogram, there is a positive smooth potential occurring on the nadir of the T wave compatible with an early afterdepolarizations (black arrowheads) followed by polyphasic potentials before a smooth potential.

on the two following beats. No EAD or EAD-like pattern is visible.

In Figure 2, a second patient with a third degree AVB has an atrial tachycardia, and a ven- tricular bigeminus. The first QRS is followed by a PVC. Its polyphasic electrogram, synchronous to the surface ECG, is preceded by a small positive smooth potential (arrows) occurring on the nadir 


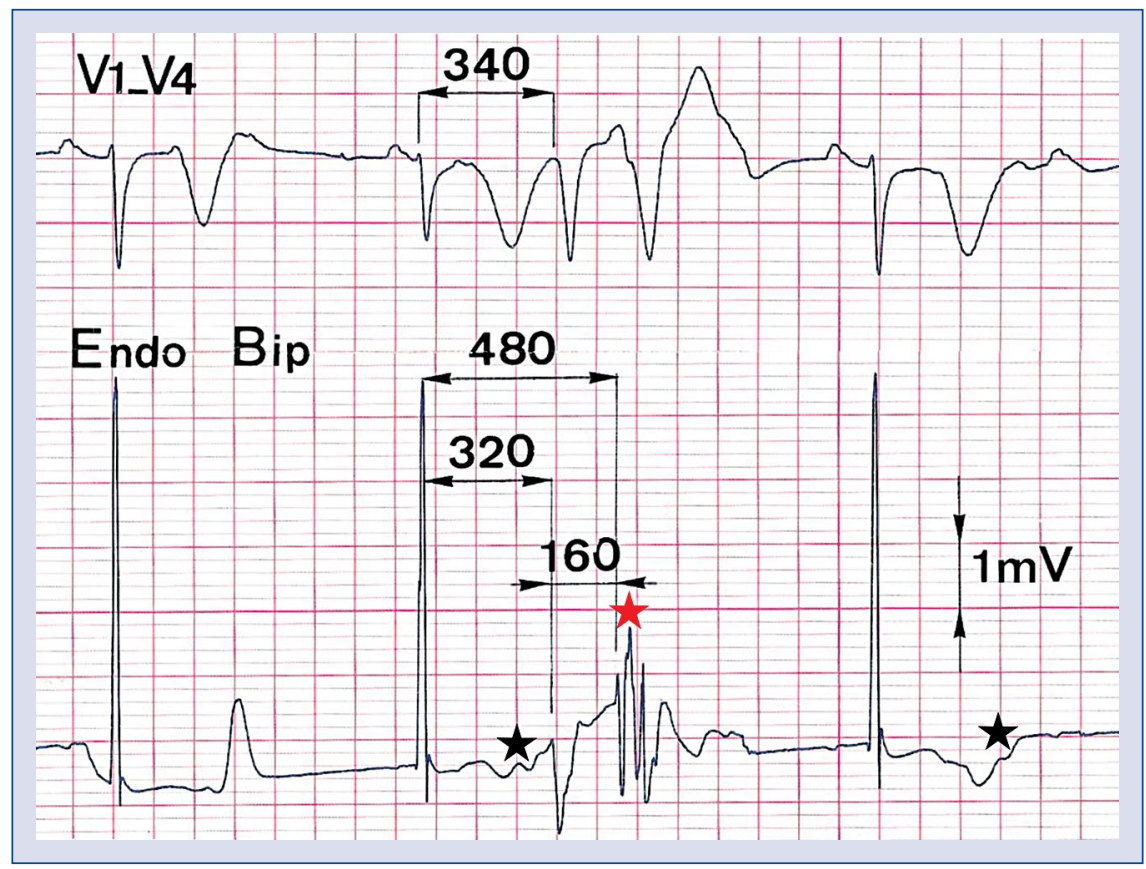

Figure 3. The second beat of a couplet is made of three consecutive sharp signals (red asterisk) with a duration of around $100 \mathrm{~ms}$. Early afterdepolarization is visible in the T wave of the endocardial signal (first black asterisk) and not on the others (second black asterisk). This figure has been published in Guy Fontaine's previous work [24].

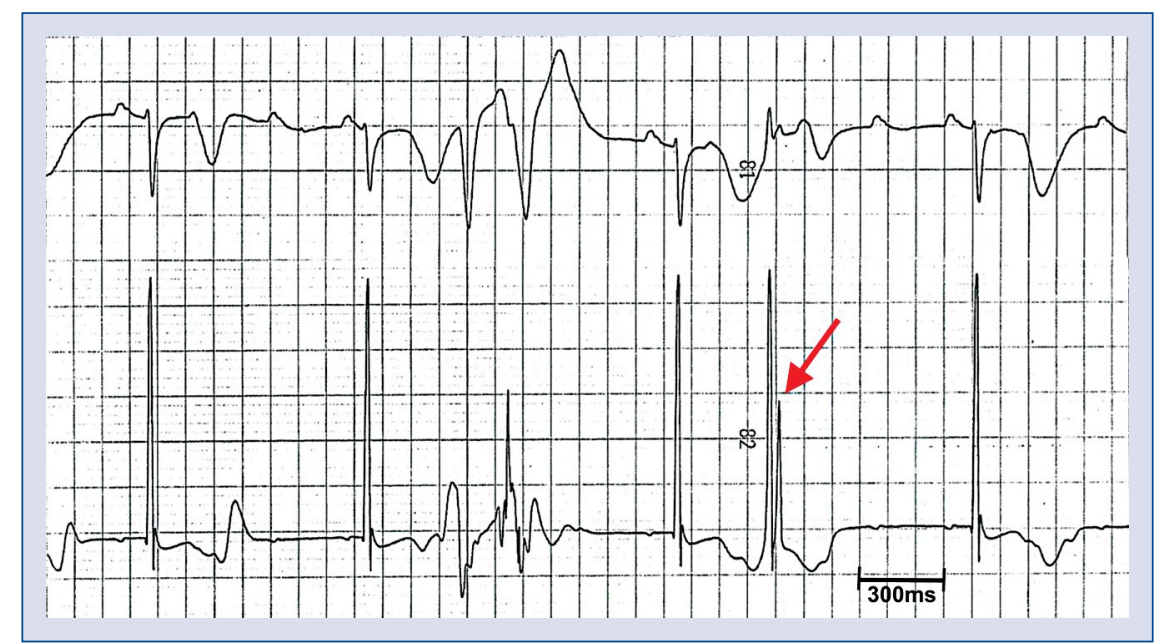

Figure 4. The same phenomenon of fragmentation with negative, positive, negative potentials with three notches on the second torsades the pointes beat with smaller amplitude is noted on this tracing. In addition, the next beat is followed by a premature ventricular contraction with double sharp positive deflections (red arrowhead) of high amplitude with no isoelectric interval in between.

of the T wave, $200 \mathrm{~ms}$ before PVC onset. It seems to have a second component, interrupted by multiphasic sharp deflections of PVC local activation and repolarization. Those smooth potentials could be part of a junctional repolarization wave, but, as they do not appear when the junctional complex is not followed by a PVC, they should reflect early depolarization activation.

Figure 3 (same patient as in Figures 2 and 4) shows a ventricular couplet. Its first beat is also preceded by a low amplitude signal synchronous to the nadir of the $\mathrm{T}$ wave as in Figure 2 (first black 


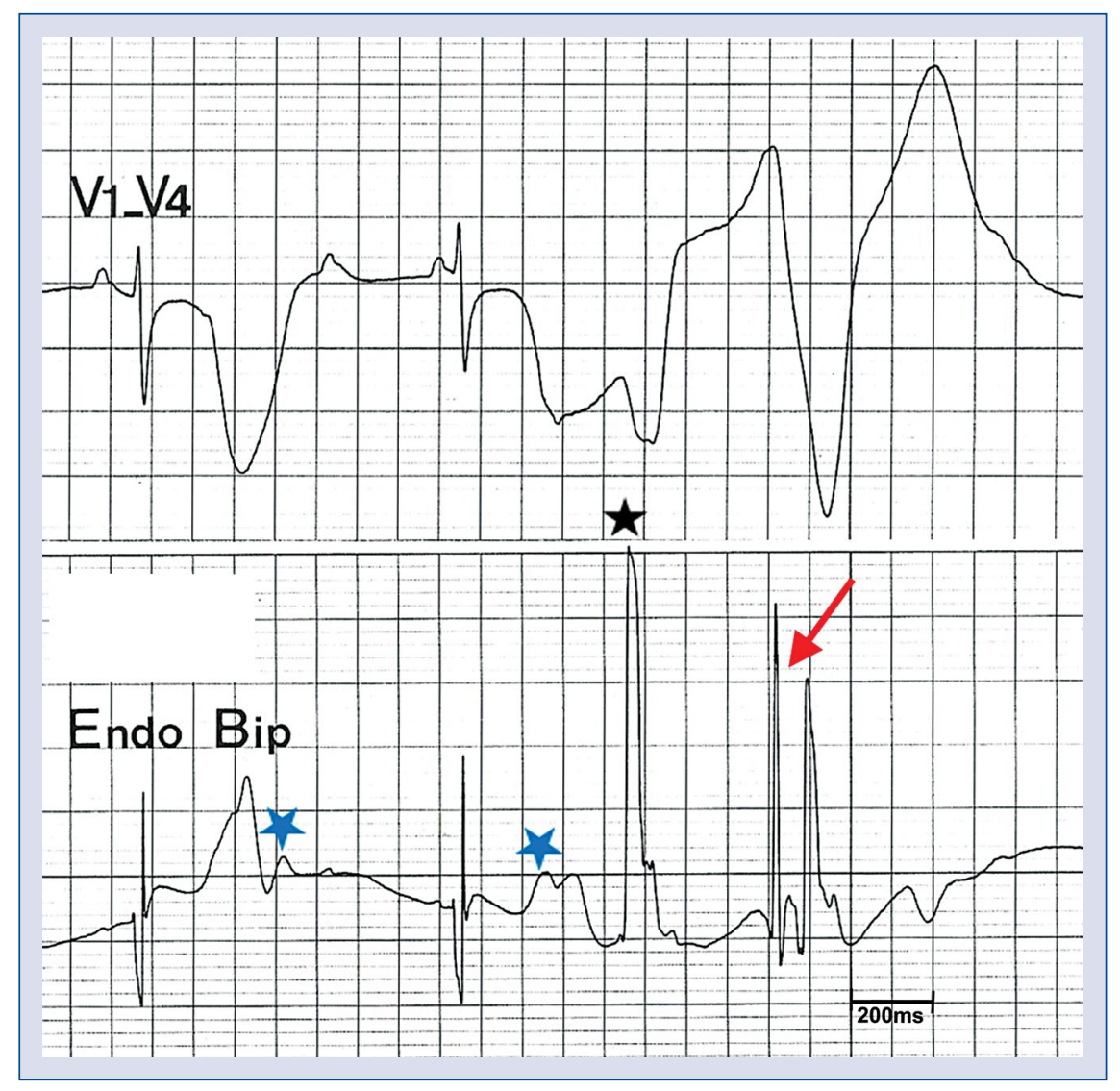

Figure 5. The same pattern with $40 \mathrm{~ms}$ interval between the two sharp potentials (red arrowhead) of high amplitude is illustrated on this tracing. This is preceded by a huge potential of the first torsades the pointes beat (black asterisk). The two junctional beats show a deflection compatible with early afterdepolarizations (blue asterisk).

star), and not on other single junctional QRS complexes. That first PVC has an endocardial morphology somehow different from the ones on Figure 2, suggesting a different origin. The following PVC is made of three consecutive sharp endocardial signals with a duration of around $100 \mathrm{~ms}$. It is interesting to note that the endocardial signals of the couplet start $20 \mathrm{~ms}$ prior to the onset of the surface QRS, indicating an origin or an exit of the $\mathrm{TdP}$ in proximity to the RV apex, where a bipolar catheter is positioned.

In the same patient, Figure 4 illustrates another ventricular couplet with the same phenomenon of fragmentation with negative, positive, negative deflections of the endocardial signal with three notches on the second PVC. In addition, the next junctional complex is followed by a single PVC with double sharp positive deflections of high amplitude with almost no isoelectric interval in between (red arrow). The isolated junctional beats again exhibited different major repolarization trouble.
Figure 5 illustrates in a third patient a similar endocardial activation pattern when compared to Figure 4 with an interval of $40 \mathrm{~ms}$ between the two sharp potentials of high amplitude (red arrow). This is preceded by a huge potential of the first $\mathrm{TdP}$ beat (black asterisk). The T waves of the two junctional beats show a deflection compatible with EADs.

Figure 6 (the same patient as Fig. 1) shows that the first beat is the basic rhythm in high degree AVB with a long QT interval showing the typical pattern of smooth and sharp potentials on the endocardial tracing. The first TdP beat exhibits a sharp notch, which is not compatible with an EAD (first arrow). The corresponding electrogram (EGM) is exactly in synchrony with the notch also not compatible with an $\mathrm{EAD}$, but suggests that the TdP starts, or has its exit in the proximity of the RV apex, since the RV endocardial signal starts approximately $20 \mathrm{~ms}$ prior to the surface ECG. The negative terminal part of the first EGM of the TdP has a letter V shape of $100 \mathrm{~ms}$ duration followed by 


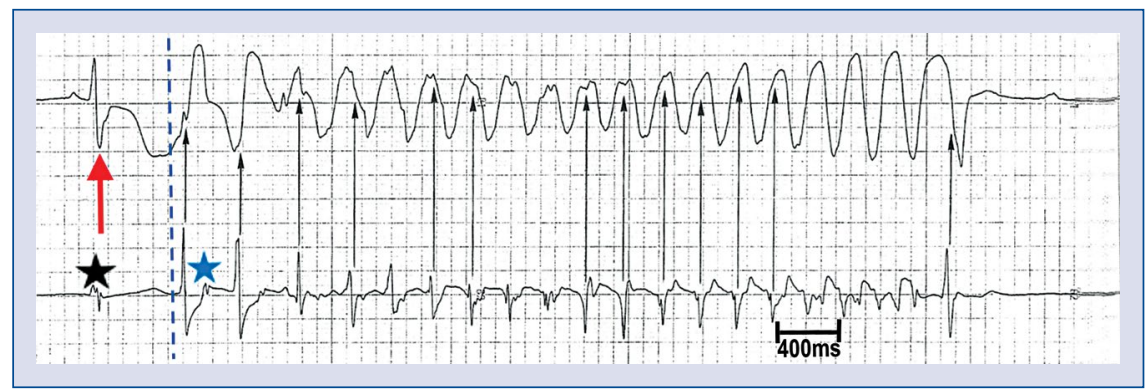

Figure 6. This tracing was the same patient as Figure 1. The endocardial signal below the surface electrocardiogram (ECG) tracing was noted. The first beat (red arrow) is the basic rhythm in high degree atrioventricular block with a long QT interval showing the typical pattern of smooth and sharp potentials. Note that the endocardial signal starts prior to the surface ECG (blue dashed line). The following beats show the narrowing of the electrograms except for the last beats, which show a fragmentation close to ventricular fibrillation. Some of the arrows stress that the local right ventricle activation can occur in diastole between two torsade de pointes beats.

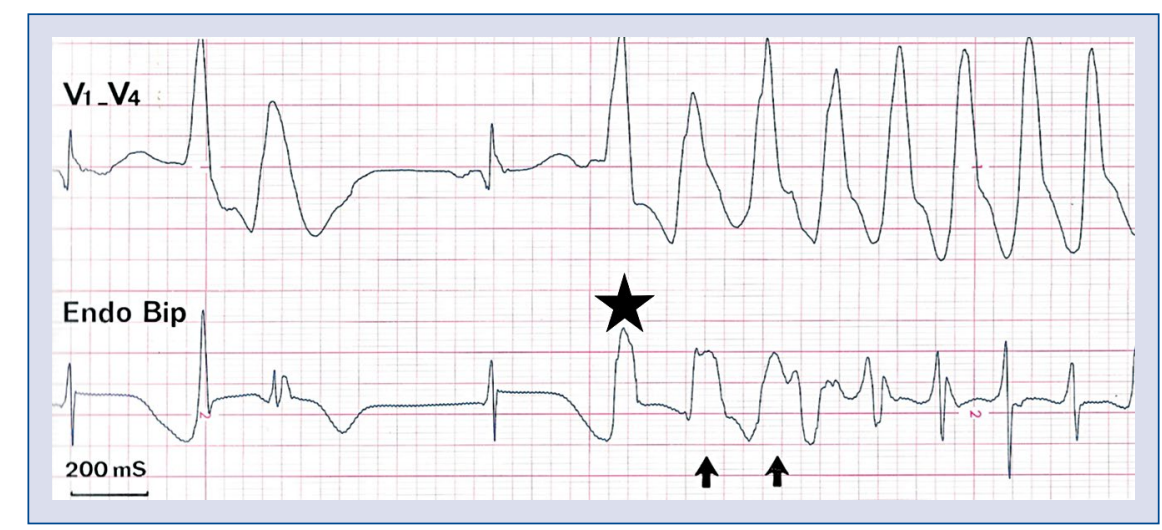

Figure 7. The tracing shows at the initiation of torsade de pointes a similar pattern such as in Figure 6 with even longer electrograms (arrows) with a reverse U-shape T wave up to $200 \mathrm{~ms}$ (black asterisk). This figure has been published in Guy Fontaine's previous work [24].

a small sharp notch. This again demonstrates the delayed activation of several contiguous structures also observed on the next TdP beat. The following beats show the narrowing of the EGMs except for the last beats, which show a fragmentation resembling ventricular fibrillation (VF). Some of the black arrows stress that RV activation can occur during diastole between two TdP beats.

The tracings of Figure 7 show that at the initiation of TdP a similar pattern of Figure 6 with even larger EGMs with an inverted "U" pattern of 200 ms (black asterisk). This is followed by narrowing of the EGM close to the basic AVB rhythm also located in the diastole between two TdP beats.

\section{Discussion}

Early afterdepolarizations (EADs) have been extensively studied in animal models and by com- puter simulation $[4,15-18]$. They are an important cause of ventricular arrhythmias in the LQTS, but the mechanisms by which EADs at the cellular scale cause arrhythmias such as TdP are unclear.

This work, which is an extension of a preliminary study previously published by the present group [14], reports on endocardial signals during episodes of TdP in high-degree AVB in humans in vivo. Endocardial signals derived from the RV apex exhibit a spectrum of fragmentations occurring on a single or multiple beats prior to the initiation of TdP, which are intermittent, transient, or irregular suggesting parallel activation of uncoupled contiguous structures in proximity to the RV apex and also demonstrate that there is a major impairment of electrotonic forces. In fact, the bipolar recording V1-V4 used in this study extracts information mostly from the RV since both electrodes are put in V1 and V4, in 
a parasternal anterior location mostly covering the RV. Indeed, this recording underscores the present hypothesis that the origin of these PVCs leading to TdP are from the RV.

In the early $60 \mathrm{~s}$ experimental animal studies from the Gordon Moe laboratory showed that prolongation of the monophasic action potential duration was time-dependent and the same group also demonstrated time-dispersion dependence of refractory periods $[19,20]$. These seminal observations, which demonstrated heterogeneities, are crucial to explain a phenomenon of phase-2 reentry, which is valid only for the first TdP beat (the second beat occurs after a short coupling interval). Multipolar plunge electrodes led to construction of a 3-dimensional (3D) isochronal map demonstrating an origin in the subendocardial tissue followed by bifurcation of activation towards the right as well as the left ventricle and the septum [21].

The present results provide new insights into the mechanism of TdP in AVB stressing the presence of a spectrum of fragmentation of endocardial signals, which can be observed on the first TdP beat, the second, or during a period of up to $200 \mathrm{~ms}$. The $\mathrm{V}$ shape or inverted U shape of endocardial signals presented at the beginning of the TdP in Figure 6 represent the most impressive pattern of activation of multiple contiguous structures. Note that it was possible to record this phenomenon without distortion because of the band-pass of the chain of apparatus including the recorders, which were going from $\mathrm{DC}$ to $60 \mathrm{~Hz}$. All these aspects demonstrate intraventricular troubles in conduction, most likely independent of an anatomical obstacle.

On all tracings of patients studied herein, the first endocardial signal occurs at the same time or prior to the onset of the QRS complex on the surface tracing. This highly suggests that in humans the origin of activation or exit site during AVB-induced TdP starts in the proximity of the RV apex, although a more septal or even left-sided origin cannot be ruled out since there were no catheters in place at these sites [21]. This is also in agreement with the work of Birati et al. [22]. It may be speculated that the human RV is thin enough to enable TdP, ideally a structure of less than its normal thickness of $3 \mathrm{~mm}$. This led to the suspicion that RV crista supraventricularis, the thinnest structure in the ventricles, could be an ideal site for initiation of TdP [23]. Study of this anatomical region by the present group in 3 patients without cardiac disease showed a thickness of crista supraventricular of $1 \mathrm{~mm}$. With this result in mind, it is now possible to interpret the fragmentation of potentials as the result of transient electrical uncoupling of contiguous structures close to RV crista supraventricularis. However, this hypothesis has to be confirmed in future 3D electroanatomical endocardial and epicardial mapping studies during $\mathrm{TdP}$ in humans with high degree AVB. Therefore, the safety factor of transmission of activation is weak producing a spectrum of intermittent, irregular, intramyocardial blocks. If this situation involves both ventricles it may explain that most TdP episodes are self-terminating and explain why they rarely degenerate into VF.

Early afterdepolarizations are secondary voltage depolarizations producing a low amplitude hump at the end of the phase- 2 of the action potential. The presence of potentials compatible with an EAD phenomenon was observed in 2 patients, and was absent in 3 . This absence can be explained by the genesis of $\mathrm{EAD}$ in a zone located far from the bipolar recording system because of heterogeneity. It is assumed that a bunch of 100 to 1000 EAD cells are necessary to have enough electrical force to produce the first PVC. However, induction of $\mathrm{TdP}$ could also be the result of a different phenomenon not involving EADs, but a local conduction disorder leading to a microreentry as suggested by this study.

\section{Limitations of the study}

This was a small study performed several decades ago, and some clinical information was missing. With today's techniques such as electroanatomical voltage mapping and modern mapping catheters, a more profound knowledge on the pathophysiology of $\mathrm{TdP}$ may be acquired in humans in vivo. Therefore, these results to some extent remain hypothetical and have to be confirmed and extended in future studies. Yet, these kind of studies in humans, where pacing is stopped in order to induce TdP nowadays has become almost impossible due to ethical restrictions. Furthermore, fragmented potentials in the initiating phase of TdP may be a bystander phenomenon, just representing conduction delay due to the short coupling intervals of PVCs. Yet, it is interesting to see that this phenomenon often occurred prior to PVC crescendo and prior to initiation of TdP. A way to exclude a causal role would be to ablate these regions and to see, whether TdP is rendered non-inducible.

\section{Conclusions}

This work demonstrated fragmented endocardial signals in the RV apical region in vivo during episodes 
of TdP in humans with high-degree AVB, and exhibits a spectrum of fragmentations occurring on a single or multiple beats prior to initiation of TdP. These fragmentations indicate areas of poor conduction and various degrees of intramyocardial block, and therefore suggest a new mechanism of $\mathrm{TdP}$ tachycardia in patients with spontaneous high-degree AVB.

\section{Acknowledgements}

We extend our gratitude to Dr. Li Zhang from the Lankenau Hospital, Philadelphia, PA, USA for her valuable remarks concerning EADs in the long QT syndrome. This study was supported by the Clinical Research Award of the First Affiliated Hospital of Xi'an Jiaotong University (XJTU/1AFCRF-2018-015; 2018MS-12).

\section{Conflict of interest: None declared}

\section{References}

1. Subbiah RN, Gollob MH, Gula LJ, et al. Torsades de pointes during complete atrioventricular block: Genetic factors and electrocardiogram correlates. Can J Cardiol. 2010; 26(4): 208-212, indexed in Pubmed: 20386770.

2. Varkevisser R, Vos MA, Beekman JD, et al. AV-block and conduction slowing prevail over TdP arrhythmias in the methoxaminesensitized pro-arrhythmic rabbit model. J Cardiovasc Electrophysiol. 2015; 26(1): 82-89, doi: 10.1111/jce.12533, indexed in Pubmed: 25154623.

3. Cho MS, Nam GB, Kim YG, et al. Electrocardiographic predictors of bradycardia-induced torsades de pointes in patients with acquired atrioventricular block. Heart Rhythm. 2015; 12(3): 498-505, doi: 10.1016/j.hrthm.2014.11.018, indexed in Pubmed: 25460857.

4. Boulaksil M, Jungschleger JG, Antoons G, et al. Drug-induced torsade de pointes arrhythmias in the chronic AV block dog are perpetuated by focal activity. Circ Arrhythm Electrophysiol. 2011; 4(4): 566-576, doi: 10.1161/CIRCEP.110.958991, indexed in Pubmed: 21622813.

5. Vandersickel N, Bossu A, De Neve J, et al. Short-lasting episodes of torsade de pointes in the chronic atrioventricular block dog model have a focal mechanism, while longer-lasting episodes are maintained by re-entry. JACC Clin Electrophysiol. 2017; 3(13): 1565-1576, doi:10.1016/j.jacep.2017.06.016, indexed in Pubmed: 29759839.

6. Smith WM, Gallagher JJ. "Les torsades de pointes": an unusual ventricular arrhythmia. Ann Intern Med. 1980; 93(4): 578-584, indexed in Pubmed:7001975.

7. Roden DM. Clinical practice. Long-QT syndrome. N Engl J Med. 2008; 358(2): 169-176, doi: 10.1056/NEJMcp0706513, indexed in Pubmed: 18184962.

8. Liu JF, Jons C, Moss AJ, et al. International Long QT Syndrome Registry. Risk factors for recurrent syncope and subsequent fatal or near-fatal events in children and adolescents with long QT syndrome. J Am Coll Cardiol. 2011; 57(8): 941-950, doi: 10.1016/j.jacc.2010.10.025, indexed in Pubmed:21329841.

9. Schwartz PJ, Woosley RL. Predicting the Unpredictable: DrugInduced QT Prolongation and Torsades de Pointes. J Am Coll Cardiol. 2016; 67(13): 1639-1650, doi: 10.1016/j.jacc.2015.12.063, indexed in Pubmed: 27150690.

10. Brugada P, Wellens HJ. Early afterdepolarizations: role in conduction block, "prolonged repolarization-dependent reexcitation," and tachyarrhythmias in the human heart. Pacing Clin Electrophysiol. 1985; 8(6): 889-896, indexed in Pubmed: 2415942.

11. Dessertenne F. [Ventricular tachycardia with 2 variable opposing foci]. Arch Mal Coeur Vaiss. 1966; 59(2): 263-272, indexed in Pubmed: 4956181.

12. D'Alnoncourt CN, Zierhut W, Blüderitz B. “Torsade de pointes” tachycardia. Re-entry or focal activity? Br Heart J. 1982; 48(3): 213-216, indexed in Pubmed: 7104111.

13. Jensen G, Sigurd B, Sandoe E. Adams-Stokes seizures due to ventricular tachydysrhythmias in patients with heart block: prevalence and problems of management. Chest. 1975; 67(1): 43-48, indexed in Pubmed: 1235328.

14. Li G, Saguner AM, Akdis D, et al. Intramyocardial block in patients with atrioventricular block. J Investig Med. 2018; 66(5): 1-4, doi: 10.1136/jim-2017-000682, indexed in Pubmed: 29514866.

15. Antzelevitch C. Basic mechanisms of reentrant arrhythmias. Curr Opin Cardiol. 2001; 16(1): 1-7, indexed in Pubmed: 11124712.

16. Gilmour RF, Moïse NS. Triggered activity as a mechanism for inherited ventricular arrhythmias in German shepherd Dogs. J Am Coll Cardiol. 1996; 27(6): 1526-1533, indexed in Pubmed: 8626969.

17. Akar FG, Yan GX, Antzelevitch C, et al. Unique topographical distribution of $\mathrm{M}$ cells underlies reentrant mechanism of torsade de pointes in the long-QT syndrome. Circulation. 2002; 105(10): 1247-1253, indexed in Pubmed: 11889021.

18. Kim TY, Kunitomo Y, Pfeiffer Z, et al. Complex excitation dynamics underlie polymorphic ventricular tachycardia in a transgenic rabbit model of long QT syndrome type 1. Heart Rhythm. 2015; 12(1): 220-228, doi: 10.1016/j.hrthm.2014.10.003, indexed in Pubmed: 25285647.

19. Han J, Millet D, Chizzonitti B, et al. Temporal dispersion of recovery of excitability in atrium and ventricle as a function of heart rate. Am Heart J. 1966; 71(4): 481-487, indexed in Pubmed: 4951481.

20. Han J, Moe GK. Nonuniform recovery of excitability in ventricular muscle. Circ Res. 1964; 14: 44-60, indexed in Pubmed: 14104163

21. El-Sherif N, Caref EB, Yin H, et al. The electrophysiological mechanism of ventricular arrhythmias in the long QT syndrome. Tridimensional mapping of activation and recovery patterns. Circ Res. 1996; 79(3): 474-492, indexed in Pubmed: 8781481.

22. Birati EY, Belhassen B, Bardai A, et al. The site of origin of torsade de pointes. Heart. 2011; 97(20): 1650-1654, doi: 10.1136/ hrt.2010.212381, indexed in Pubmed: 21561894.

23. James TN. Anatomy of the crista supraventricularis: its importance for understanding right ventricular function, right ventricular infarction and related conditions. J Am Coll Cardiol. 1985; 6(5): 1083-1095, indexed in Pubmed: 2931470.

24. Fontaine G. A new look of torsades de pointes. Ann N Y Acad Sci. 1992; 644: 157-177. 